Article

\title{
Long-Term Hydropower Generation Scheduling of Large-Scale Cascade Reservoirs Using Chaotic Adaptive Multi-Objective Bat Algorithm
}

\author{
Lyuwen Su${ }^{\circledR}$, Kan Yang * ${ }^{\mathbb{D}}, \mathrm{Hu} \mathrm{Hu}$ and Zhe Yang \\ College of Hydrology and Water Resources, Hohai University, Nanjing 210098, China; lwsu@hhu.edu.cn (L.S.); \\ huhu619485776@hhu.edu.cn (H.H.); zyang7279@hhu.edu.cn (Z.Y.) \\ * Correspondence: kyang@hhu.edu.cn; Tel.: +86-1305-750-2351
}

Received: 25 October 2019; Accepted: 11 November 2019; Published: 13 November 2019

\begin{abstract}
With growing concerns over renewable energy, the cascade hydropower reservoirs operation (CHRO), which balances the development of economic benefits and power supply security, plays an increasingly important role in hydropower systems. Due to conflicting objectives and complicated operation constraints, the $\mathrm{CHRO}$ problem considering the requirements of maximizing power generation benefit and firm power output is determined as a multi-objective optimization problem (MOP). In this paper, a chaotic adaptive multi-objective bat algorithm (CAMOBA) is proposed to solve the CHRO problem, and the external archive set is added to preserve non-dominant solutions. Meanwhile, population initialization based on the improved logical mapping function is adopted to improve population diversity. Furthermore, the self-adaptive local search strategy and mutation operation are designed to escape local minima. The CAMOBA is applied to the CHRO problem of the Qingjiang cascade hydropower stations in southern China. The results show that CAMOBA outperforms the multi-objective bat algorithm (MOBA) and non-dominated sorting genetic algorithms-II (NSGA-II) in different hydrological years. The spacing (SP) and hypervolume (HV) metrics verify the excellent performance of CAMOBA in diversity and convergence. In summary, the CAMOBA is demonstrated to get better scheduling solutions, providing an effective approach for solving the cascade hydropower reservoirs operation (CHRO).
\end{abstract}

Keywords: cascade hydropower reservoirs operation; firm power output; multi-objective bat algorithm; Qingjiang

\section{Introduction}

In recent years, with the increasing awareness of environmental protection and sustainable development, renewable energy has attracted more and more people's attention [1]. Hydropower generated by fast running or falling water has become an important part of renewable energy [2,3]. The total generation of hydropower in China exceeded 1 trillion $\mathrm{kWh}$ by the end of 2015, while the total installed capacity of hydropower reached 320 million kilowatts [4]. Therefore, the operation of cascade hydropower stations is becoming an important issue in the optimization of power systems in China. Generally, properly increasing the output of hydropower stations in dry seasons is of great significance to meet the load balance of power systems and improve the safety of power supplies. However, increasing the output in dry seasons will cause the water level to drop faster. The loss of water head will reduce the total annual generating capacity of the power station. Therefore, in order to meet actual requirements of the cascade hydropower reservoirs operation $(\mathrm{CHRO})$ problem, this paper establishes the reservoir operation model of cascade hydropower stations with consideration of annual power generation benefit and firm power output. 
Obviously, the CHRO problem of power generation benefit and firm power output is extended to a multi-objective optimization problem (MOP) with two conflicting objectives. In the past few decades, researchers have made many efforts to handle the MOP problem using the modern heuristic multi-objective evolutionary algorithm (MOEA). Algorithms such as the multi-objective genetic algorithm (MOGA) [5,6], multi-objective particle swarm optimization (MOPSO) [7], multi-objective harmony search (MOHS) [8], multi-objective ant colony optimization (MOACO) [9], the multi-objective evolutionary algorithm based on decomposition (MOEA/D) [10] and non-dominated sorting genetic algorithms-II (NSGA-II) [11] have become increasingly prominent in methods for addressing various aspects of MOP issues.

The bat algorithm (BA) is a new heuristic algorithm based on microbats echolocation behavior [12], which integrates the advantages of particle swarm optimization (PSO), harmony search (HS) and the simulated annealing algorithm (SA). As a promising evolutionary algorithm, BA can effectively solve the problem of high nonlinearity and is easy to implement [12]. Case studies include micro-grid operation management [13], reservoir operation [14], interconnected power systems [15], dynamic economic dispatch [16] and others. In 2011, the BA, originally developed solving single-objective optimization problems (SOP), was modified by Yang [17] for multi-objective optimization problems (MOP). In the multi-objective bat algorithm (MOBA), all objectives are merged into one objective by a weighted sum, and the weights are generated randomly from the uniform distribution. Many scholars put forward some improved methods and applied them in many fields. Yang [18] proposes a modified multi-objective bat algorithm, adding inertia weight into the velocity updating equation to enhance the convergence of the algorithm. The results show that the proposed method is feasible and effective for solving passive power filter (PPF) design problems. Aiming at solving the combined economic/emission dispatch problem, Liang [19] proposed a multi-objective hybrid bat algorithm. The modified comprehensive learning strategy and random black hole model are introduced to overcome drawbacks of MOBA. The new multi-objective self-adaptive learning bat-inspired algorithm (SALBA) was proposed by Niknam [16] to solve practical reserve constrained dynamic economic dispatch. A novel self-adaptive learning method is used in SALBA to increase the population and modify the convergence criterion. Bansal [20] proposed a novel parallel hybrid multi-objective bat algorithm (PHMOBA) for solving multi-objective engineering design problems, and concludes that PHMOBA has a better convergence and success rate than MOBA. However, there are some limitation in MOBA and the proposed algorithms: (1) When the MOP problem has a non-convex Pareto optimal front, the weighted sum method cannot obtain Pareto optimal solutions. Meanwhile, the uniform distribution of the weight vector may not obtain the Pareto optimal solution with uniform distribution in most nonlinear MOP problems [21]. So, the weighted sum method is not the best way to solve MOP problems; (2) the initial population is generated randomly in MOBA, which may lead to repeated solutions occupying memory spaces, and the initial solution may be concentrated in a limited space; (3) algorithms may converge prematurely and fall into a local solution; (4) a few scholars extend the MOBA to the CHRO problem, especially for the large-scale reservoir system. Therefore, this paper proposes a chaotic adaptive multi-objective bat algorithm (CAMOBA) and applies it to solve the CHRO problem. In CAMOBA, (1) the external archive set $E A S()$ is added to preserve the non-dominant solutions found during evolution. The crowding distance is used to maintain the size of the external archive set; (2) the population initialization based on chaos theory is adopted to improve population diversity; (3) the self-adaptive local search strategy based on the normal cloud model is proposed to update solutions. The mutation operation is designed to mitigate the premature convergence and jump out of local solutions.

This paper is organized as follows. The mathematical modeling of the CHRO problem is introduced in Section 2. The standard bat algorithm and multi-objective bat algorithm are described in Section 3. Section 4 demonstrates the details of the proposed chaotic adaptive multi-objective bat algorithm for solving the $\mathrm{CHRO}$ problem. Section 5 reports the application in Qingjiang River and 
the comparison between results of the proposed method and other algorithms. In the end, Section 6 outlines the conclusions of this work.

\section{Mathematical Modeling of the CHRO Problem}

\subsection{Objective Function}

A multi-objective model is developed to optimize the $\mathrm{CHRO}$ problem that needs to meet two objective functions during scheduling.

(1) Maximizing the annual power generation of the hydropower system:

$$
f_{1}=\max \sum_{k=1}^{K} \sum_{t=1}^{T} N_{k, t} \times \Delta t=\max \sum_{k=1}^{K} \sum_{t=1}^{T} L_{k} \times Q_{k, t}^{L E C} \times H_{k, t} \times \Delta t
$$

(2) Maximizing the firm power output:

$$
f_{2}=\max _{1 \leq t \leq T}\left\{\min \sum_{k=1}^{K} N_{k, t}\right\}
$$

where $N_{k, t}$ is the power output of the $k$ th reservoir at $t$ th period; $L_{k}$ is the synthetic output coefficient of the $k$ th reservoir; $H_{k, t}$ is the hydraulic head of the $k$ th reservoir at the $t$ th period $(\mathrm{m})$; $Q_{k, t}^{L E C}$ is the generation flow of the $k$ th reservoir at the $t$ th period $\left(\mathrm{m}^{3} / \mathrm{s}\right) ; \Delta t$ is the time duration of a single period.

\subsection{Constraints}

Objective functions are subject to the following constraints when solving the $\mathrm{CHRO}$ problem.

(1) Reservoir water balance equation:

$$
S V_{k, t}=S V_{k, t-1}+\left(I_{k, t}-Q_{k, t}^{\text {OUT }}\right) \times \Delta t k=1,2, \cdots, K, t=1,2, \cdots, T
$$

(2) Reservoir storage conversion:

$$
S V_{k, t}=s v_{k}\left(Z_{k, t}\right) k=1,2, \cdots, K, t=1,2, \cdots, T+1
$$

(3) Reservoir water head:

$$
\begin{gathered}
Z_{k, t}=s v_{k}^{-1}\left[\left(s V_{k, t}+S V_{k, t-1}\right) / 2\right] k=1,2, \cdots, K, t=1,2, \cdots, T \\
Z_{k, t}^{\text {down }}=z_{k}\left(Q_{k, t}^{\text {OUT }}\right) k=1,2, \cdots, K, t=1,2, \cdots, T \\
H_{k, t}=Z_{k, t}-Z_{k, t}^{\text {down }} k=1,2, \cdots, K, t=1,2, \cdots, T
\end{gathered}
$$

(4) Reservoir water level constraint:

$$
Z_{k, t}^{\min } \leq Z_{k, t} \leq Z_{k, t}^{\max } k=1,2, \cdots, K, t=1,2, \cdots, T+1
$$

(5) Reservoir outflow constraint:

$$
\begin{aligned}
& Q_{k, t}^{\text {OUT }}=Q_{k, t}^{L E C}+Q_{k, t}^{S} k=1,2, \cdots, K, t=1,2, \cdots, T \\
& Q_{k, t}^{\text {min }} \leq Q_{k, t}^{\text {OUT }} \leq Q_{k, t}^{\max } k=1,2, \cdots, K, t=1,2, \cdots, T
\end{aligned}
$$




$$
Q_{k, t}^{\max }=q_{k}\left(Z_{k, t}\right) k=1,2, \cdots, K, t=1,2, \cdots, T
$$

(6) Power output constraint:

$$
N_{k, t} \leq N_{k, t}^{\max } k=1,2, \cdots, K, t=1,2, \cdots, T
$$

where $S V_{k, t}$ and $S V_{k, t-1}$ are storage capacity of the $k$ th reservoir at the $t$ th and $(t-1)$ th period, respectively $\left(\mathrm{m}^{3}\right) ; I_{k, t}$ and $Q_{k, t}^{\text {OUT }}$ are inflow and outflow of the $k$ th reservoir at the $t$ th period, respectively $\left(\mathrm{m}^{3} / \mathrm{s}\right) ; Z_{k, t}$ is the water level of the $k$ th reservoir at the $t$ th period $(\mathrm{m}) ; s v_{k}\left(Z_{k, t}\right)$ is the storage-capacity curve of the $k$ th reservoir; $z_{k}\left(Q_{k, t}^{\text {OUT }}\right)$ is the function between the outflow and tail water level of the $k$ th reservoir; $Z_{k, t}^{\text {down }}$ is the tail water level of the $k$ th reservoir $(\mathrm{m}) ; Z_{k, t}^{\min }$ and $Z_{k, t}^{\max }$ are the lowest and highest water level of the $k$ th reservoir at the $t$ th period, respectively (m); $Q_{k, t}^{\text {OUT }}$ is the outflow of the $k$ th reservoir at the $t$ th period $\left(\mathrm{m}^{3} / \mathrm{s}\right) ; Q_{k, t}^{S}$ is the spillage of the $k$ th reservoir at the $t$ th period $\left(\mathrm{m}^{3} / \mathrm{s}\right) ; Q_{k, t}^{\min }$ and $Q_{k, t}^{\max }$ are minimum and maximum outflow of the $k$ th reservoir at the $t$ th period, respectively $\left(\mathrm{m}^{3} / \mathrm{s}\right) ; q_{k}\left(Z_{k, t}\right)$ is the function between the water level and maximum outflow of the $k$ th reservoir; $N_{k, t}^{\max }$ is the maximum power output of the $k$ th reservoir at the $t$ th period.

\section{Overview of the Multi-Objective Bat Algorithm}

The bat algorithm (BA) is a new heuristic algorithm based on the microbat echolocation behavior and proposed by Yang in 2010 [12]. In order to simplify and facilitate application, the algorithm adopts three idealized conditions: (1) The bat uses the echolocation principle to confirm the distance and accurately distinguish between obstacles and prey; (2) bats fly with speed $V_{j}$ and a fixed frequency $F_{\min }$ to search for prey at coordinate position (solution) $X_{j}$. They can flexibly amend the frequency $F$ and adjust the pulse emission rate $r_{j}$ based on their distance from the target; (3) assume the loudness $A_{j}$ changing from a maximum value to a small value.

In the BA, new solutions and velocity updates can be obtained from Equations (13) and (14).

$$
\begin{gathered}
V_{j}^{g}=V_{j}^{g-1}+\left(X_{j}^{g}-X_{*}\right) \times\left(F_{\min }+\left(F_{\max }-F_{\min }\right) \beta\right) \\
X_{j}^{g}=X_{j}^{g-1}+V_{j}^{g}
\end{gathered}
$$

where $\beta \in[0,1]$ is a random vector; $j$ is the number of bats; $g$ is the iteration number; $X_{*}$ is the current global best location; $F \in\left[F_{\min }, F_{\max }\right]$ is the frequency; $V_{j}^{g}$ is the velocity of the $j$ th bat in the $g$ th generation; $X_{j}^{g}$ is the position (solution) of the $j$ th bat in the $g$ th generation.

For each bat, if rand $>r_{j}$, the new solution $X_{j, \text { new }}^{g}$ is generated around the current best solution to complete the local search according to the Equation (15).

$$
X_{j, \text { new }}^{g}=X_{j, \text { old }}^{g}+\varepsilon \times \overline{A^{g}}
$$

where $X_{j, o l d}^{g}$ is the best solution of the $j$ th bat in the first to $g$ th generation; $X_{j, \text { new }}^{g}$ is the new solution of the $j$ th bat in the $g$ th generation; $\varepsilon \in[-1,1]$ is a random vector; $\overline{A^{g}}$ is the mean loudness of all the bats in the gth generation.

If rand $>A_{j}$ and the fitness of $X_{j, \text { new }}^{g}$ is better than that of $X_{*}, X_{*}$ is replaced by $X_{j, \text { new }}^{g}$. Then reduce $A_{j}$ and increase $r_{j}$ according to Equations (16) and (17).

$$
\begin{gathered}
A_{j}^{g+1}=\alpha \times A_{j}^{g} \\
r_{j}^{g+1}=r_{j}^{1} \times[1-\exp (-\gamma \times g)]
\end{gathered}
$$


where $\alpha$ and $\gamma$ are constants; $A_{j}^{g+1}$ is the loudness of the $j$ th bat in $g+1$ th generation; $r_{j}^{g+1}$ is the pulse emission rate of the $j$ th bat in the $g+1$ th generation; $g$ is the iteration number.

In 2011, Yang proposed the MOBA algorithm based on BA to solve multi-objective optimization problems. The weighted sum is used to combine all objectives $f_{m}$ into a single objective in MOBA according to Equation (18) [17].

$$
f=\sum_{m=1}^{M} \omega_{m} \times f_{m}, \sum_{m=1}^{M} \omega_{m}=1
$$

where $\omega_{m}$ is the weight of the $m$ th objective. All weights are generated randomly from the uniform distribution $(0,1)$.

\section{Implementation of CAMOBA for solving the CHRO problem}

Assuming that the $D$ dimensional real space is the search space of the optimization problem, the algorithm is a generation population $R^{g}=\left\{X_{1}^{g}, X_{2}^{g}, \ldots, X_{j}^{g}, \cdots, X_{N G}^{g}\right\}^{T}$ composed of NG D dimensional real parameter vectors $X_{j}^{g}=\left\{x_{j, 1}^{g}, x_{j, 2}^{g}, \cdots, x_{j, d}^{g} \cdots, x_{j, D}^{g}\right\}$. Where $j$ is the number of individuals in the population, $j=1,2, \cdots, N G$; $g$ is the iteration number, $g=1,2, \cdots, g_{\max }$. In the CHRO problem, $D$ is the number of operation periods. The water levels of all reservoirs are chosen as the decision variable and encoded. The solution $X_{j}^{g}$ is shown as follows.

$$
X_{j}^{g}=\left[\begin{array}{cccc}
Z_{1,1}^{g} & Z_{1,2}^{g} & \cdots & Z_{1, T}^{g} \\
Z_{2,1}^{g} & Z_{2,2}^{g} & \cdots & Z_{2, T}^{g} \\
\cdots & \cdots & Z_{k, t}^{g} & \cdots \\
Z_{K, 1}^{g} & Z_{K, 2}^{g} & \cdots & Z_{K, T}^{g}
\end{array}\right]
$$

where $Z_{k, t}^{g}$ is the water level of the $k$ th reservoir at the $t$ th period in the $g$ th generation $(\mathrm{m})$.

\subsection{External Archive Set Maintenance and Updating Operation}

Zitzler [22] proposed an external population (called an archive set) in 1999 to preserve the non-dominant solutions found during evolution. The external archive set is added and named EAS( ) in this paper. The size of the external archive set is generally a constant $N_{E}$ due to the limitation of the computation source. The following strategies are used to incorporate all non-dominant solutions found in the current population $g$ (called CSet $(g)$ ) into $E A S\left(\right.$ ): For each solution $n_{g, j}$ in CSet $(g)$ :

(1) If $E A S\left(\right.$ ) is vacant, put $n_{g, j}$ into $E A S($ ) directly.

(2) Compare $n_{g, j}$ with other solutions in $E A S\left(\right.$ ). If $n_{g, j}$ is not dominated by any solution in $E A S($ ), add $n_{g, j}$ into $E A S$ ( ) and delete the solution dominated by $n_{g, j}$ in $E A S($ ).

(3) If the number of individuals in $E A S\left(\right.$ ) exceeds the $N_{E}$, a crowding distance comparison is performed for each new solution addition. In this process, the solution with the smallest crowding distance is eliminated. Non-dominated solutions are ranked based on one of the objective functions. The boundary solutions (the smallest and largest solutions of the function values) 
are given a larger distance value to maintain the diversity of the external archive set [23]. The crowding distance can be expressed as:

$$
\text { Crowd }_{j}=\left\{\begin{array}{c}
\sum_{m=1}^{M}\left(\frac{F i t_{1}^{m}-F i t_{2}^{m}}{\max \left\{F i t^{m}\right\}-\min \left\{F i^{m}\right\}}\right)^{2} * 100 \quad j=1 \\
\sum_{m=1}^{M}\left(\frac{F i t_{j-1}^{m}-F i t_{j+1}^{m}}{\max \left\{F i t^{m}\right\}-\min \left\{F i t^{m}\right\}}\right)^{2} j \in\left[2, N_{E}\right] \\
\sum_{m=1}^{M}\left(\frac{F i i_{N_{E}}^{m}-F i t_{N_{E}+1}^{m}}{\max \left\{F i t^{m}\right\}-\min \left\{F t^{m}\right\}}\right)^{2} * 100 j=N_{E}+1
\end{array}\right.
$$

where Crowd $_{j}$ is the crowding distance of the $j$ th solution; Fit $_{j}^{m}$ is the fitness of the $j$ th solution of the $m$ th objective; $\min \left\{F i t^{m}\right\}$ and $\max \left\{F i t^{m}\right\}$ are the minimum and maximum fitness of the $m$ th objective, respectively.

\subsection{Initial Population Generation}

The diversity and distribution of the initial population can influence the final optimal solutions of the algorithm. In MOBA, the initial population is usually generated randomly, which may lead to repeated solutions occupying memory space, and initial solutions may be concentrated in a limited space (Figure 1a). In recent years, chaotic sequences have gradually replaced random sequences and achieved good results in many applications due to nonlinear phenomena such as ergodicity, randomness and certainty of chaos [24]. Thus, the initial population is generated based on chaos theory to enhance distribution diversity and uniformity of the population (Figure 1b). There are many models to generate chaotic sequences, and the improved logical mapping function [25] is adopted in this paper to generate the chaotic sequences. Its equation is as follows:

$$
y_{d}^{q+1}=1-2 \times\left(y_{d}^{q}\right)^{2} d=1,2 \cdots, D ; q=1,2 \cdots, q_{\max }
$$

where $y_{d}^{q}$ is a chaotic variable and $y_{d}^{q} \in(-1,1)$. Equation (21) is chaotic state when $y_{d}^{1} \neq 0$.

(a) MOBA

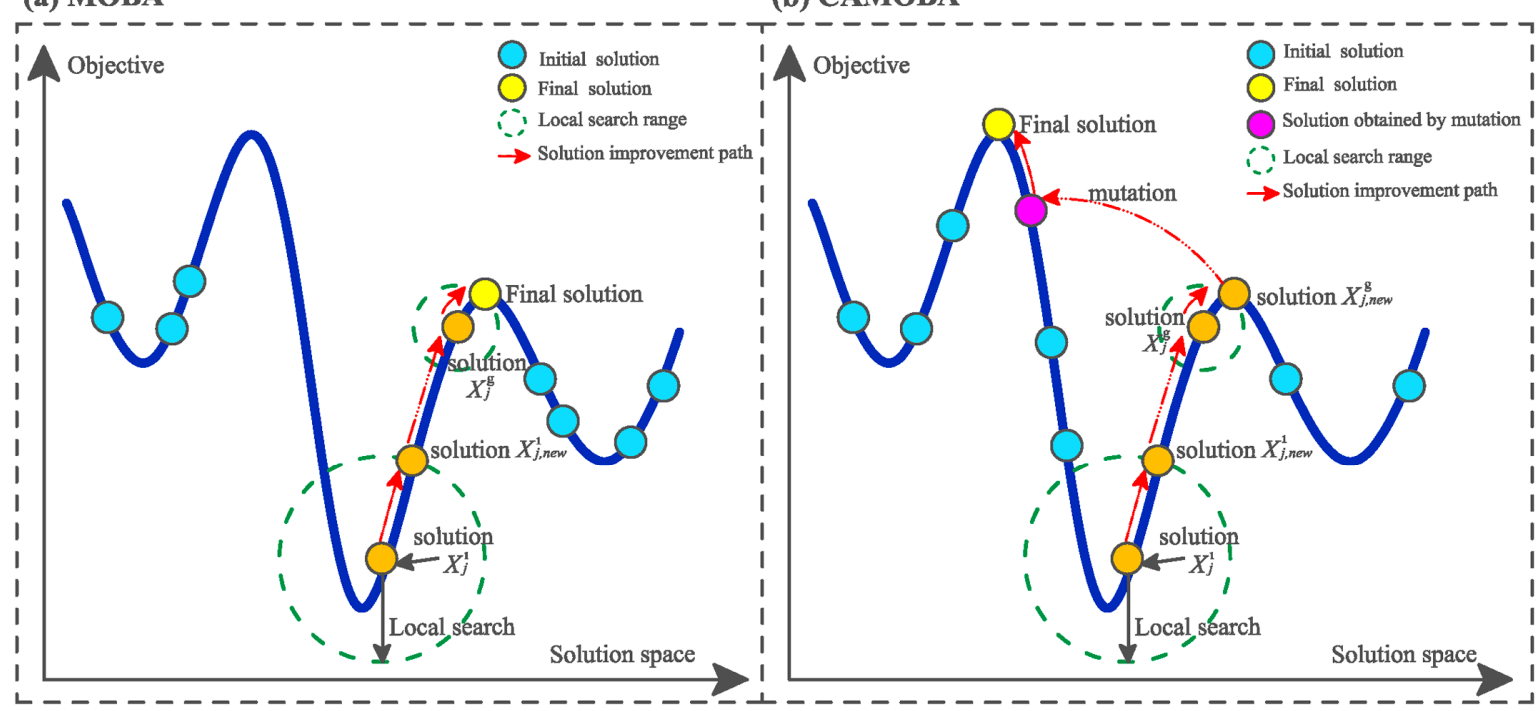

Figure 1. Sketch map of optimization processes: (a) Multi-objective bat algorithm (MOBA); and (b) chaotic adaptive multi-objective bat algorithm (CAMOBA). 
Generate $D$ different initial values, and then generate $D$ chaotic sequences with different trajectories by iteration using Equation (21). Convert chaotic variables $y_{d}^{q}$ to the value interval of the decision variables $\left[x_{d}^{\min }, x_{d}^{\max }\right]$, and initial positions $x_{j, d}^{1}$ of the bat algorithm are generated by Equation (22).

$$
x_{j, d}^{1}=x_{d}^{\min }+\left(x_{d}^{\max }-x_{d}^{\min }\right) \cdot y_{d}^{q} j=q
$$

where $x_{d}^{\max }$ and $x_{d}^{\min }$ are the maximum and minimum value of the $d$ th decision variable, respectively; $x_{j, d}^{1}$ is the initial position of the $j$ th bat in the $d$ th dimension.

\subsection{Self-Adaptive Local Search Strategy}

Due to its properties of stable and randomness tendency, the normal cloud model [26] is integrated into CAMOBA to maintain the diversity of solutions. Each cloud is determined by three main parameters: Entropy parameter $E_{n}$, expectation parameter $E_{x}$ and hyper entropy parameter $H_{e}$. The cloud operator $L\left(E_{x}, E_{n}, H e\right)$ of the cloud model for the current best solution is formulated as follows:

$$
\left\{\begin{array}{c}
E_{x}=X_{j, o l d}^{g} \\
E_{n}=3 A^{g} / 2 \\
H_{e}=E_{n} / 10
\end{array}\right.
$$

where, $E_{x}, E_{n}$ and $H_{e}$ are the expectation value, entropy and hyper-entropy, respectively.

Steps of cloud drops generation are given as follows:

Step 1: Generate a random normal distribution value $E_{n}^{\prime}$ with the expected value of $E_{n}$ and hyper entropy $H_{e}$.

Step 2: Generate a random normal distribution value $x_{p}$ with the expected value of $E_{x}$ and hyper entropy $E_{n}^{\prime}$ generated in step 1.

Step 3: Calculate the membership degree $u_{p}$, where $u_{p}=e^{\frac{-\left(x_{p}-E_{x}\right)^{2}}{2\left(E_{n}^{\prime}\right)^{2}}}$.

Step 4: One drop $\left(x_{p}, u_{p}\right)$ is attained. Steps 1 to 3 are repeated until enough cloud drops are generated.

Step 5: Each cloud drop represents a potential solution. If the newly generated cloud drop (bat) dominates the current best solution $X_{j, o l d}^{g}$ the $X_{j, o l d}^{g}$ will be replaced.

The self-adaptive local search strategy is described as in Equation (24). In order to judge whether the bat individual is in a stable state, two variables stable $(g)$ and move $(g)$ are introduced. The stable state is defined as: Bat individual $j$ satisfies stable $(g)<10^{-6} \&$ move $(g)<10^{-6}$ in successive $0.05 g_{\max }$ generations.

$$
\begin{gathered}
X_{j, \text { new }}^{g}=\left\{\begin{array}{r}
X_{j, \text { old }}^{g}+\varepsilon \times \overline{A^{g}} \text { bat individual } j \text { is in the move state } \\
\text { cloud drop bat individual } j \text { is in the stable state }
\end{array}\right. \\
\left\{\begin{array}{l}
\operatorname{stable}(g)=a b s\left(f\left(X_{j, \text { old }}^{g}\right)-f\left(X_{j, \text { old }}^{*}\right)\right) \\
\operatorname{move}(g)=\operatorname{abs}\left(f\left(X_{j, \text { old }}^{g}\right)-f\left(X_{j, \text { old }}^{g-1}\right)\right)
\end{array}\right.
\end{gathered}
$$

where, $f\left(X_{j, \text { old }}^{g}\right)$ is the fitness of bat individual $j ; f\left(X_{j, \text { old }}^{*}\right)$ is the optimal fitness of bat individual $j$ in iteration; stable $(g)$ is the gap between the current fitness of the $j$ th bat and the optimal fitness in iteration; move $(g)$ is the fitness increment of the $j$ th bat in the $g$ th generation; $g_{\max }$ is the maximum iteration. 


\subsection{Mutation Operation}

In the multi-objective bat algorithm (MOBA), the decreased diversity may cause precocity convergence (Figure 1a). Therefore, the mutation operation, as shown in Figure 1b, is proposed to diversify bats and enhance the ability of the global search, which can be expressed as:

$$
X_{j, E A S}^{g}=X_{R_{1}, E A S}^{g}+\delta\left(X_{R_{2}, E A S}^{g}-X_{R_{3}, E A S}^{g}\right)
$$

where, $X_{R_{1}}^{g}, E A S^{\prime} X_{R_{2}, E A S}^{g}$ and $X_{R_{3}}^{g}$,EAS are three archive individuals randomly chosen from the external archival set $E A S() . \delta$ is the mutation constant that is used to regulate the size of the disturbance and enhance the performance of the bat. Then, the bat $X_{j, E A S}^{g}$ is compared with other solutions in EAS( ) to update the $E A S($ ).

\subsection{Procedures of CAMOBA for Solving the CHRO Problem}

The flowchart of CAMOBA for solving the CHRO problem is presented in Figure 2.

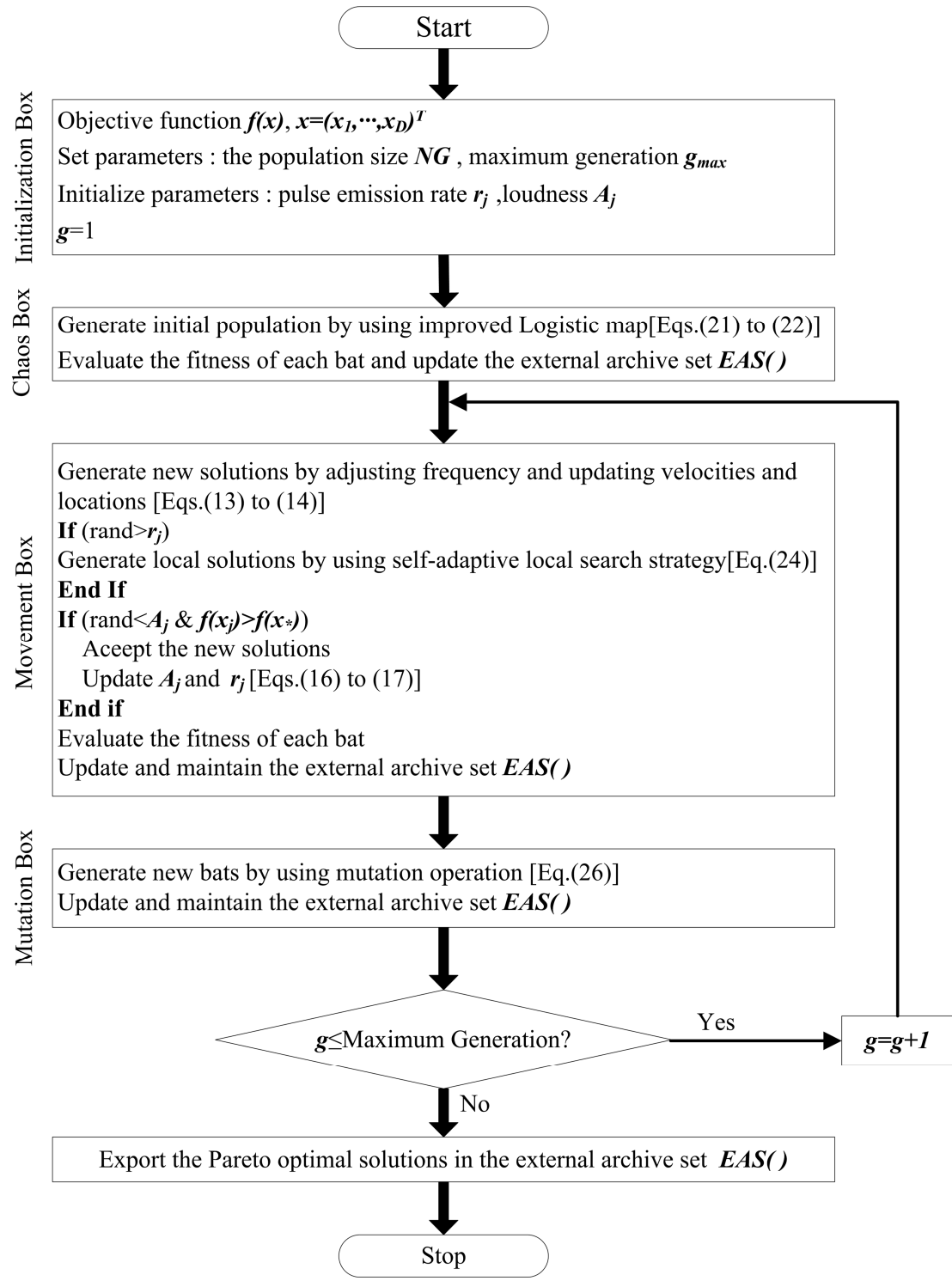

Figure 2. Flowchart of CAMOBA for solving the cascade hydropower reservoirs operation (CHRO) problem. 


\section{Case Study}

\subsection{Case Study Description}

The three hydropower stations on the Qingiiang River in China are used to verify the practical feasibility of CAMOBA for solving the CHRO problem. The Qingjiang River is the first main tributary of the Yangtze River below the Three Gorges. The $423 \mathrm{~km}$ long Qingjiang River has a catchment area of $1.7 \times 10^{3} \mathrm{~km}^{2}$. The Qingjiang cascade hydropower project plays an important role in promoting social and economic development of the Yangtze River with multiple functions, including hydropower generation, flood control, ecological protection, etc. Figure 3 shows a diagram of the Yangtze River Basin and locations of the study area in the Qingjiang River. In this study, some basic constraints are set as follows. The upper water level bounds of the Shuibuya Reservoir, Geheyan Reservoir and Gaobazhou Reservoir in the flood season (from June to July) are set to $391.8 \mathrm{~m}, 193.6 \mathrm{~m}$ and $78.5 \mathrm{~m}$, respectively. Meanwhile, during the non-flood season, the water level of the Shuibuya Reservoir, Geheyan Reservoir and Gaobazhou Reservoir cannot exceed the normal water levels of 400 m, $200 \mathrm{~m}$ and $80 \mathrm{~m}$, respectively. The lower water level bounds of the three cascade reservoirs all year are $350 \mathrm{~m}$, $160 \mathrm{~m}$ and $78 \mathrm{~m}$, respectively. The Shuibuya Reservoir, Geheyan Reservoir and Gaobazhou Reservoir are installed with capacities of $1840 \mathrm{MW}, 1210 \mathrm{MW}$ and $270 \mathrm{MW}$, respectively.

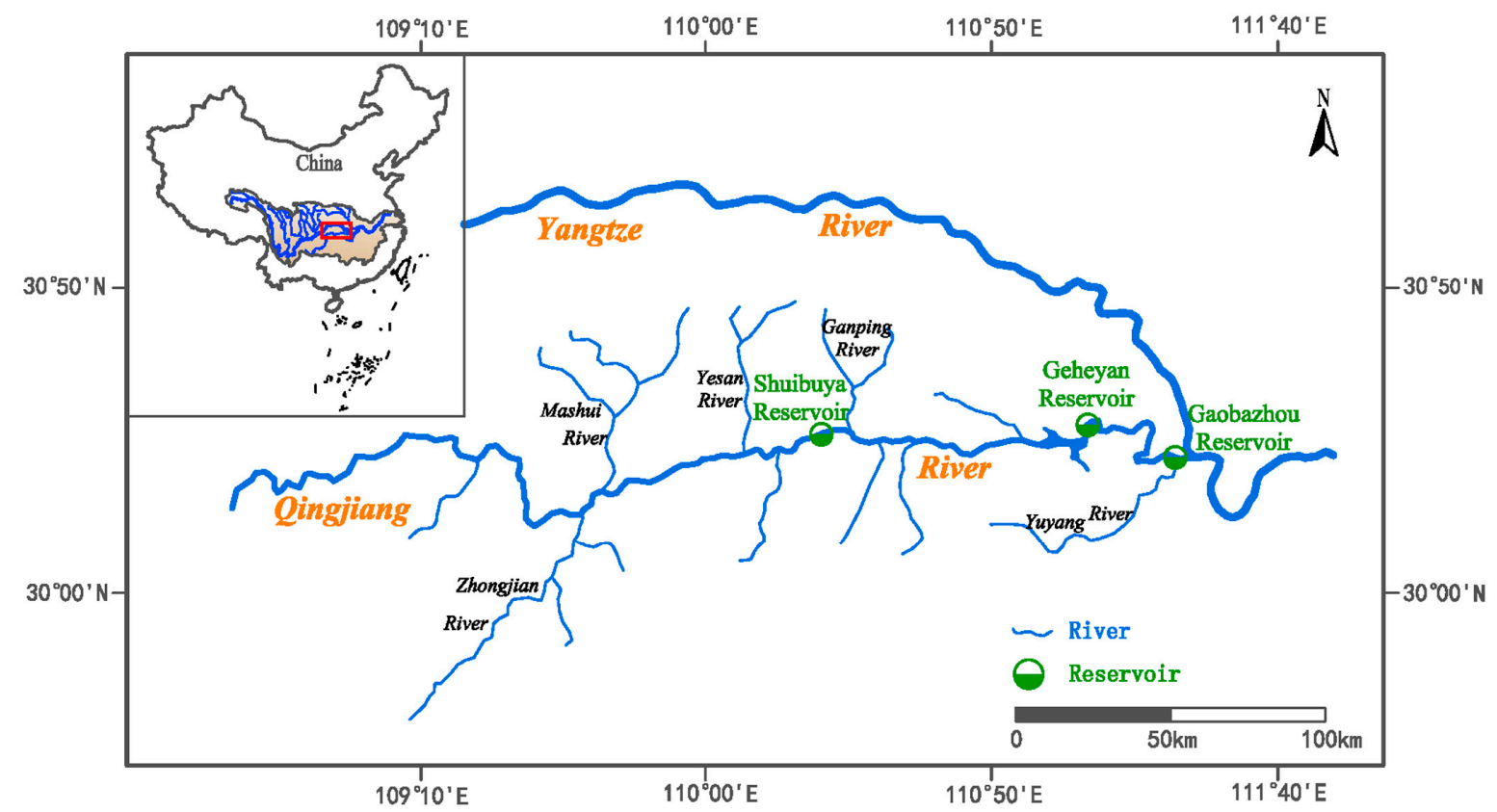

Figure 3. The location of cascade reservoirs in the Qingjiang River.

The hydrological year can be divided into three types, wet, normal and dry years, according to the annual runoff [27]. According to the runoff data of the Qingjiang River basin from 1971 to 2006, the typical representatives of June 1995 to May 1996, June 2004 to May 2005 and June 1985 to May 1986 are selected as the wet, normal and dry years, respectively. The whole operation time is divided into 12 periods, each of which is one month. The monthly inflow data of the Qingjiang cascade reservoirs in these three typical years are shown in Figure 4. 


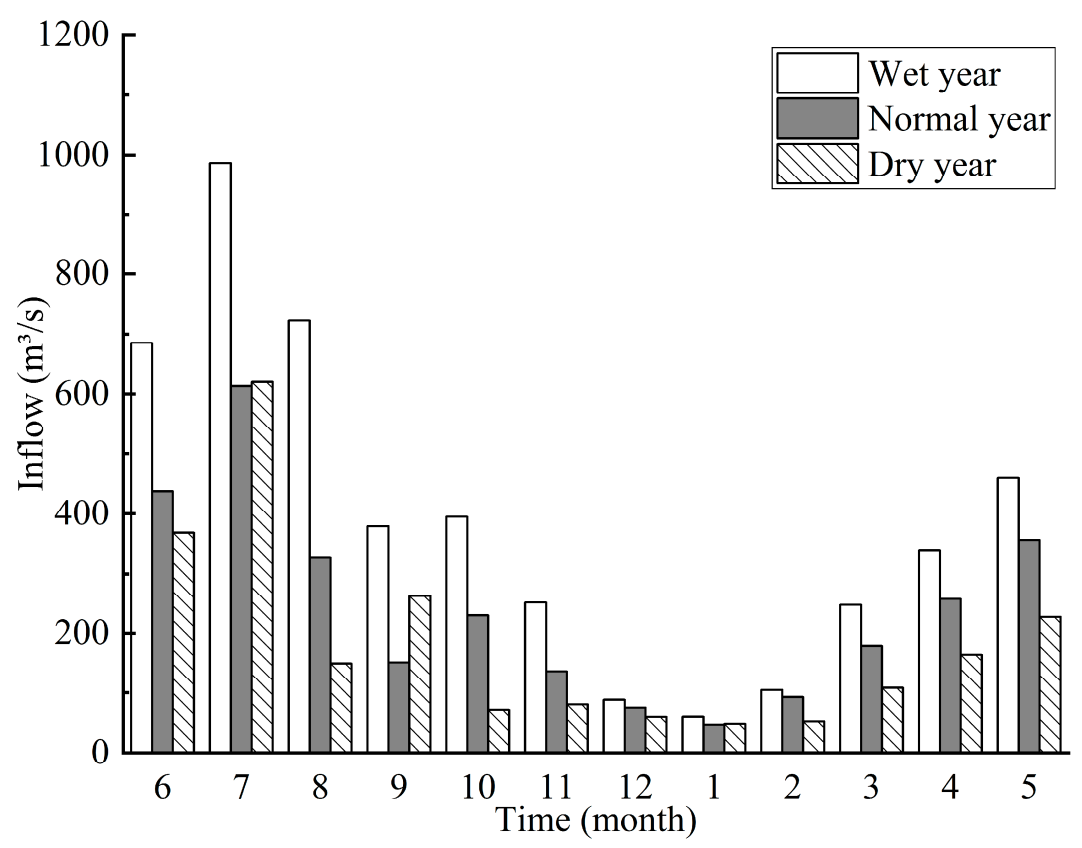

Figure 4. Inflow of a hydropower system in three different hydrological years.

\subsection{Parameter Settings}

To testify the feasibility and effectiveness of CAMOBA in the application of the CHRO problem, CAMOBA is compared with MOBA [17] and NSGA-II [24]. NSGA-II is a high-performance multi-objective evolutionary algorithm that has been widely used in various disciplines [28]. The three optimization methods perform 10 independent runs. The maximum iteration $g_{\max }$ is set at 1000 for the three algorithms. For CAMOBA and NSGA-II, the population size NG and the external archive set size $N_{E}$ are set at 200 and 30. For MOBA, the population size $N G$ is 30 . The maximum iteration of the chaos operator $q_{\max }$ is 200 in CAMOBA. The mutation constant $\delta$ is 0.1 in CAMOBA. The other parameters of CAMOBA and MOBA are taken from reference [17]. The frequency $F$ is varied from 0 to $1 ; \alpha$ and $\gamma$ are set at 0.9. The parameters of NSGA-II take the recommended values specified in reference [29]; the probabilities of crossover and mutation are set at 0.8 and 0.33 , respectively.

\subsection{Results and Discussion}

The Pareto optimal fronts of different hydrological years calculated by the three methods are drawn in Figure 5. It can be seen from Figure 5 that the CHRO problem can be solved by CAMOBA, MOBA and NSGA-II. Meanwhile, it can be clearly observed that there is a competitive relationship between two targets in wet, normal and dry years, and the greater the annual power generation, the smaller the firm power output. The annual power generation in a wet year is larger than that in a normal year, and the annual power generation in a dry year is the smallest. Moreover, it can be easily concluded that when solving the complex CHRO problem by NSGA-II and MOBA, the resulting Pareto solution is dominated by CAMOBA, which shows that CAMOBA can generate more annual power generation with the same firm power output. For example, when the maximal firm power output is considered, the proposed CAMOBA method can increase the firm power output by about $21.1423 \mathrm{MW}$ and 32.5989 MW, respectively, compared to the MOBA and NSGA-II in a normal year while increasing the annual power generation $0.0668 \times 10^{8} \mathrm{kWh}$ and $0.1551 \times 10^{8} \mathrm{kWh}$, respectively. Meanwhile, it can be seen that CAMOBA has a more widely distributed optimal solution than MOBA and NSGA-II, which means that CAMOBA performs better in terms of solution diversity. The maximum (Max), mean (Mean) and standard deviation (Std) of the maximum annual power generation and firm power output are listed in Table 1. As shown in Table 1, it can be clearly seen that solutions of CAMOBA are close to the best solution, while all the indexes of CAMOBA are better than those of MOBA and 
NSGA-II. For example, the standard deviations of CAMOBA are 0.0173 and 29.5803 with respect to two objectives and less than that of MOBA and NSGA-II in a normal year. Thus, the results show that CAMOBA can provide better scheduling solutions compared to MOBA and NSGA-II when dealing with the complex CHRO issue.
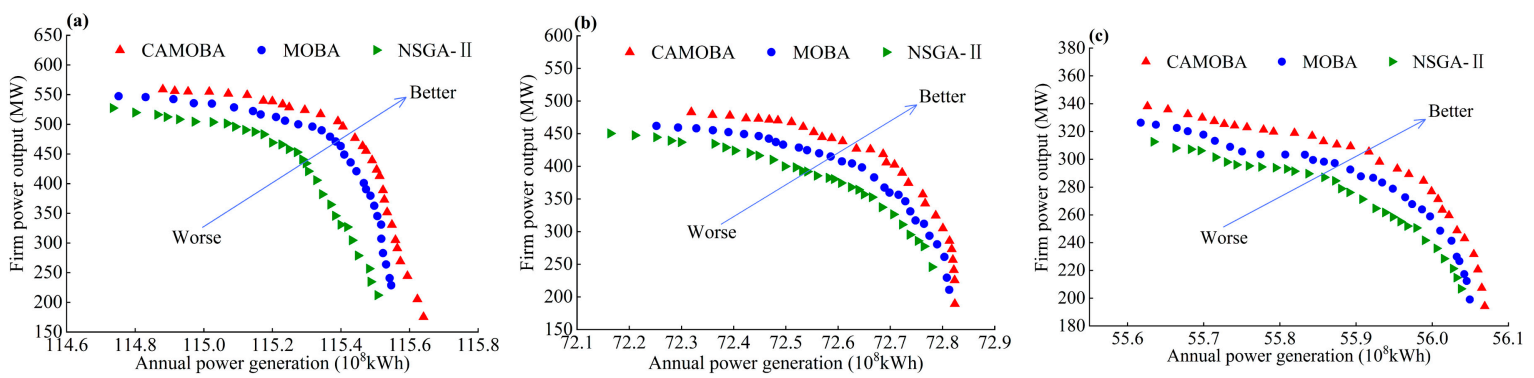

Figure 5. Pareto optimal fronts of different methods in different hydrological years: (a) Wet year; (b) normal year; and (c) dry year.

Table 1. Operation results for the best annual power generation $\left(10^{8} \mathrm{kWh}\right)$ and firm power output (MW) with 10 runs obtained by three algorithms.

\begin{tabular}{ccccccccc}
\hline $\begin{array}{c}\text { Hydrological } \\
\text { Years }\end{array}$ & Objective & Method & Max $\uparrow$ & $\begin{array}{c}\text { Improve } \\
\text { (\%) }\end{array}$ & Mean $\uparrow$ & $\begin{array}{c}\text { Improve } \\
\text { (\%) }\end{array}$ & Std $\downarrow$ & $\begin{array}{c}\text { Improve } \\
\text { (\%) }\end{array}$ \\
\hline \multirow{5}{*}{ Wet year } & Annual & CAMOBA & 115.6408 & $0.082 \%$ & 115.5407 & $0.048 \%$ & 0.0408 & $3.702 \%$ \\
& power & MOBA & 115.5694 & $0.021 \%$ & 115.4885 & $0.003 \%$ & 0.0414 & $2.172 \%$ \\
\cline { 2 - 9 } & generation & NSGA-II & 115.5456 & - & 115.4851 & - & 0.0423 & - \\
\hline \multirow{5}{*}{ Norm } & CAMOBA & 560.6503 & $3.368 \%$ & 539.3225 & $5.774 \%$ & 22.6059 & $31.829 \%$ \\
& power & MOBA & 550.5622 & $1.508 \%$ & 525.0124 & $2.967 \%$ & 26.9268 & $18.798 \%$ \\
& output & NSGA-II & 542.3833 & - & 509.8830 & - & 33.1604 & - \\
\hline & Annual & CAMOBA & 72.8236 & $0.005 \%$ & 72.8125 & $0.054 \%$ & 0.0173 & $67.544 \%$ \\
& power & MOBA & 72.8227 & $0.004 \%$ & 72.7947 & $0.029 \%$ & 0.0415 & $22.043 \%$ \\
& generation & NSGA-II & 72.8199 & - & 72.7735 & - & 0.0532 & - \\
\cline { 2 - 9 } & Firm & CAMOBA & 483.0351 & $7.237 \%$ & 431.6210 & $9.679 \%$ & 29.5803 & $19.650 \%$ \\
& power & MOBA & 461.8928 & $2.543 \%$ & 409.1474 & $3.968 \%$ & 30.5043 & $17.140 \%$ \\
& output & NSGA-II & 450.4362 & - & 393.5319 & - & 36.8142 & - \\
\hline \multirow{5}{*}{ Dry year } & Annual & CAMOBA & 56.0700 & $0.002 \%$ & 56.0621 & $0.046 \%$ & 0.0145 & $47.405 \%$ \\
& power & MOBA & 56.0697 & $0.001 \%$ & 56.0582 & $0.040 \%$ & 0.0203 & $26.570 \%$ \\
& generation & NSGA-II & 56.0690 & - & 56.0360 & - & 0.0276 & - \\
\cline { 2 - 8 } & Firm & CAMOBA & 338.1704 & $5.592 \%$ & 299.2050 & $4.398 \%$ & 20.4751 & $11.893 \%$ \\
& power & MOBA & 326.3672 & $1.906 \%$ & 288.7327 & $0.744 \%$ & 22.2445 & $4.279 \%$ \\
& output & NSGA-II & 320.2625 & - & 286.6007 & - & 23.2388 & - \\
\hline
\end{tabular}

Note: $\downarrow$ denotes that smaller value is better. $\uparrow$ means that the bigger value is better. Improvement in CAMOBA $=$ $\mid$ CAMOBA-NSGA-II $\mid /$ NSGA-II $\times 100 \%$. Improvement in MOBA $=\mid$ MOBA-NSGA-II $\mid / N S G A-I I \times 100 \%$.

The spacing (SP) [30] and hypervolume (HV) [31] metrics are used to further compare the performance in diversity and convergence of the proposed CAMOBA method with MOBA and NSGA-II. The three optimization methods perform 10 independent runs. The maximum (Max), minimum (Min), mean (Mean), standard deviation (Std) and average execution times of SP and HV are listed in Table 2, and best results are indicated in bold type. As the average computational time of the three algorithms listed in Table 2 show, CAMOBA is faster than NSGA-II but slightly slower than MOBA. CAMOBA consumes more computational time than MOBA because of the addition of the external archive set and the mutation operation to the proposed CAMOBA method. However, the primary purpose of algorithms is to obtain better Pareto optimal fronts. The running time of CAMOBA in Table 2 is reasonable and acceptable. Besides, the maximum and minimum SP results of NSGA-II are slightly better than CAMOBA in a wet year. However, the mean and standard deviation SP results of CAMOBA are significantly better than MOBA and NSGA-II in the three hydrological years. The 
observations indicate that CAMOBAs are superior to MOBA and NSGA-II in most of the runs in terms of diversity. Meanwhile, the HV results of CAMOBA are significantly better than those of MOBA and NSGA-II in all typical years, indicating that CAMOBA is superior to the other two algorithms in terms of convergence. The SP and HV convergence curves of the three algorithms are provided in Figure 6. The whole convergence process and the detailed convergence trajectory in iteration 1 to 200 are shown in the first and second rows, respectively. As can be seen from Figure 6, CAMOBA is the first one that converges to a good level and remains stable in all hydrological years. Therefore, the feasibility and superiority of the proposed CAMOBA method for solving the multi-objective CHRO problem in wet, normal and dry years within a reasonable execution time have been verified.

Table 2. Spacing (SP) and hypervolume (HV) metric values and average execution times obtained by the three algorithms in different hydrological years.

\begin{tabular}{ccccccccccc}
\hline \multirow{2}{*}{$\begin{array}{c}\text { Hydrological } \\
\text { Years }\end{array}$} & \multirow{2}{*}{ Algorithm } & \multicolumn{4}{c}{ SP } & \multicolumn{6}{c}{ HV } & \multirow{2}{*}{ Time(s) } \\
\cline { 3 - 10 } Wet year & Max $\downarrow$ & Mean $\downarrow$ & Min $\downarrow$ & Std $\downarrow$ & Max $\uparrow$ & Mean $\uparrow$ & Min $\uparrow$ & Std $\downarrow$ & \\
& CAMOBA & 0.0247 & $\mathbf{0 . 0 2 2 0}$ & 0.0190 & $\mathbf{0 . 0 0 1 7}$ & $\mathbf{0 . 8 1 8 3}$ & $\mathbf{0 . 8 1 1 4}$ & $\mathbf{0 . 7 9 4 9}$ & $\mathbf{0 . 0 0 7 5}$ & 511.2 \\
& MOBA & 0.0729 & 0.0662 & 0.0618 & 0.0042 & 0.7367 & 0.7220 & 0.7124 & 0.0085 & $\mathbf{4 6 1 . 4}$ \\
& NSGA-II & $\mathbf{0 . 0 2 4 5}$ & 0.0221 & $\mathbf{0 . 0 1 6 5}$ & 0.0028 & 0.7320 & 0.7211 & 0.7057 & 0.0093 & 759.6 \\
\hline \multirow{3}{*}{ Normal year } & CAMOBA & $\mathbf{0 . 0 1 9 4}$ & $\mathbf{0 . 0 1 4 8}$ & $\mathbf{0 . 0 1 1 2}$ & $\mathbf{0 . 0 0 2 7}$ & $\mathbf{0 . 8 1 4 7}$ & $\mathbf{0 . 8 0 3 8}$ & $\mathbf{0 . 7 8 1 2}$ & $\mathbf{0 . 0 1 0 7}$ & 515.4 \\
& MOBA & 0.0681 & 0.0626 & 0.0519 & 0.0048 & 0.7821 & 0.7449 & 0.7123 & 0.0252 & $\mathbf{4 5 6 . 8}$ \\
& NSGA-II & 0.0398 & 0.0345 & 0.0298 & 0.0039 & 0.7414 & 0.7150 & 0.6968 & 0.0158 & 741.3 \\
\hline \multirow{3}{*}{ Dry year } & CAMOBA & $\mathbf{0 . 0 2 2 3}$ & $\mathbf{0 . 0 2 0 4}$ & $\mathbf{0 . 0 1 8 7}$ & $\mathbf{0 . 0 0 1 1}$ & $\mathbf{0 . 8 9 7 9}$ & $\mathbf{0 . 8 8 5 2}$ & $\mathbf{0 . 8 7 3 2}$ & $\mathbf{0 . 0 0 8 0}$ & 509.7 \\
& MOBA & 0.1940 & 0.1748 & 0.1658 & 0.0076 & 0.8165 & 0.8007 & 0.7826 & 0.0111 & $\mathbf{4 5 7 . 6}$ \\
& NSGA-II & 0.0384 & 0.0359 & 0.0317 & 0.0023 & 0.5525 & 0.5283 & 0.5213 & 0.0094 & 753.7 \\
\hline
\end{tabular}

Note: $\downarrow$ denotes that the smaller value is better. $\uparrow$ means that the bigger value is better.

For simplicity, only the results of the normal year are presented in detail. The reservoir operation processes of the 1st, 15th and 30th schemes (Table 3) obtained by CAMOBA are displays in Figure 7. From Figure 7, it can be seen that there is significant difference between the three scheduling schemes. The main focus of the 1st scheme is maximizing annual power generation. The monthly power generation of the 1st scheme varies greatly in a year. In the dry season (from December to February), reservoirs in the 1st scheme reduce outflows to reserve water, and the power output is lower than other schemes at this time. When inflow is large in March and April, a large amount of water is discharged from reservoirs in the 1st scheme to generate more power generation than other schemes. The 30th scheme focuses on maximizing the firm power output. Reservoirs in the 30th scheme discharge more water than other schemes in the dry season to generate more power output and improve the power supply safety of the power system. It can be seen that the monthly power generation of the 30th scheme has the smallest variation in a year. The 15th scheme is a compromise between annual power generation and firm power output. It is conducive to the balanced development of economic benefits and power supply security.

Table 3. Non-dominated schemes obtained by CAMOBA in a normal year.

\begin{tabular}{ccccccccc}
\hline Scheme & $\begin{array}{c}f_{\mathbf{1}} \mathbf{( 1 0}^{\mathbf{8}} \\
\mathbf{k W h})\end{array}$ & $\boldsymbol{f}_{\mathbf{2}} \mathbf{( M W )}$ & Scheme & $\begin{array}{c}f_{\mathbf{1}} \mathbf{( 1 0}^{\mathbf{8}} \\
\mathbf{k W h})\end{array}$ & $f_{\mathbf{2}} \mathbf{( M W )}$ & Scheme & $\begin{array}{c}f_{\mathbf{1}}\left(\mathbf{1 0}^{\mathbf{8}}\right. \\
\mathbf{k W h})\end{array}$ & $f_{\mathbf{2}} \mathbf{( M W )}$ \\
\hline 1 & 72.8236 & 189.1719 & 11 & 72.7347 & 374.6256 & 21 & 72.5535 & 452.4976 \\
2 & 72.8232 & 225.4624 & 12 & 72.7227 & 390.1868 & 22 & 72.5333 & 460.2505 \\
3 & 72.8217 & 241.1590 & 13 & 72.7082 & 402.6378 & 23 & 72.5109 & 467.7229 \\
4 & 72.8200 & 256.8327 & 14 & 72.6925 & 405.8924 & 24 & 72.4848 & 470.2905 \\
5 & 72.8177 & 273.0836 & 15 & 72.6864 & 418.7940 & 25 & 72.4680 & 471.5678 \\
6 & 72.8134 & 285.7809 & 16 & 72.6620 & 425.8439 & 26 & 72.4475 & 472.9724 \\
7 & 72.8007 & 304.9514 & 17 & 72.6352 & 427.0217 & 27 & 72.4238 & 473.4131 \\
8 & 72.7871 & 324.6124 & 18 & 72.6088 & 438.4034 & 28 & 72.3926 & 477.6139 \\
9 & 72.7668 & 343.0365 & 19 & 72.5878 & 443.0313 & 29 & 72.3594 & 478.6686 \\
10 & 72.7623 & 357.0580 & 20 & 72.5694 & 444.8507 & 30 & 72.3189 & 483.0351 \\
\hline
\end{tabular}


(a)
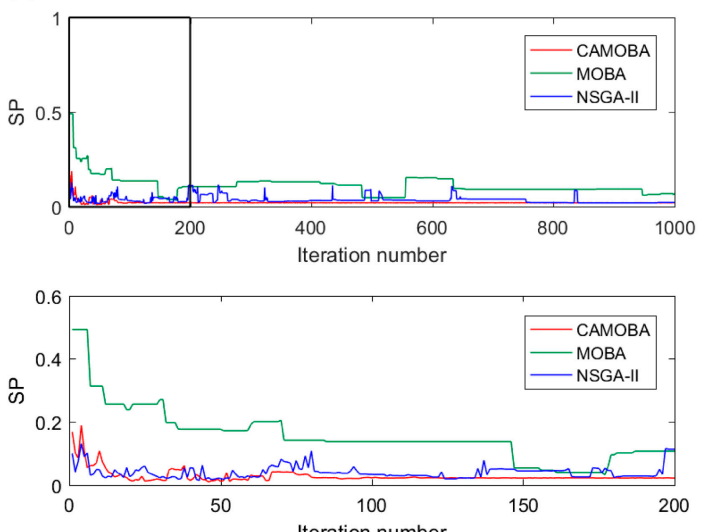

(c)
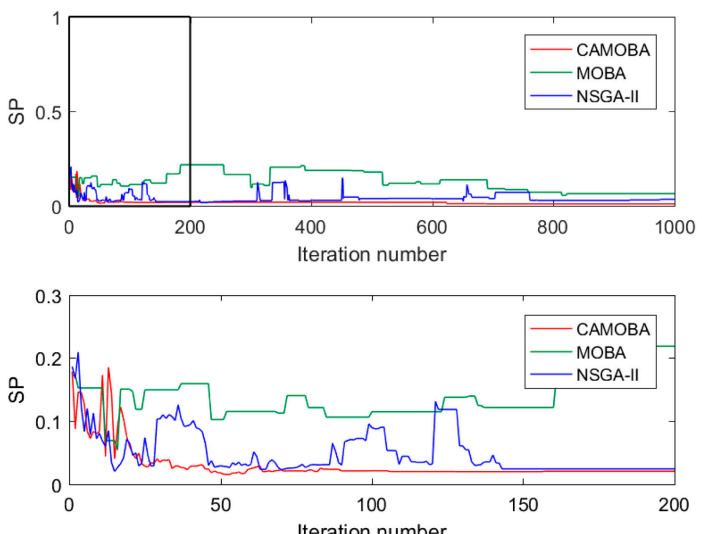

(e)
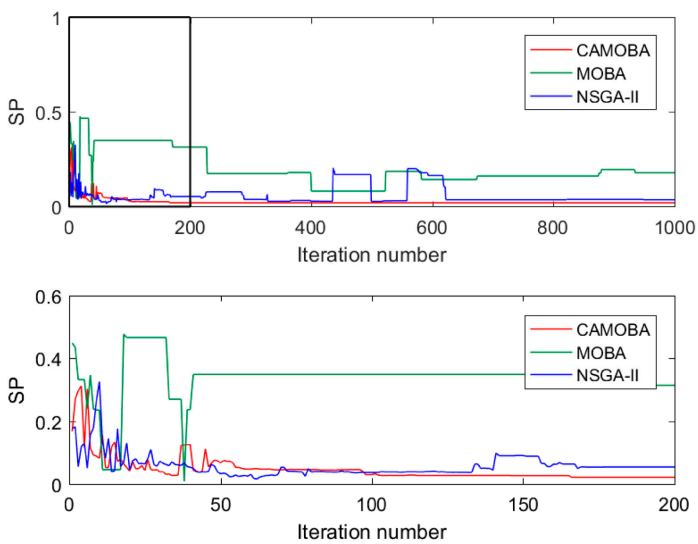

(b)
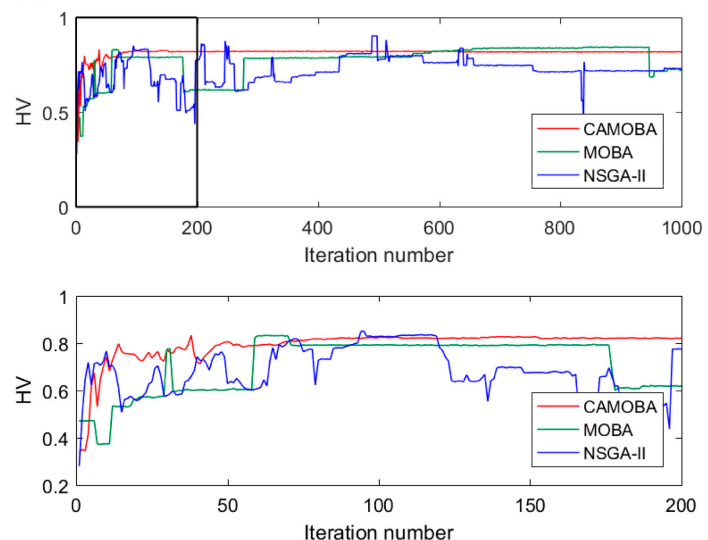

(d)
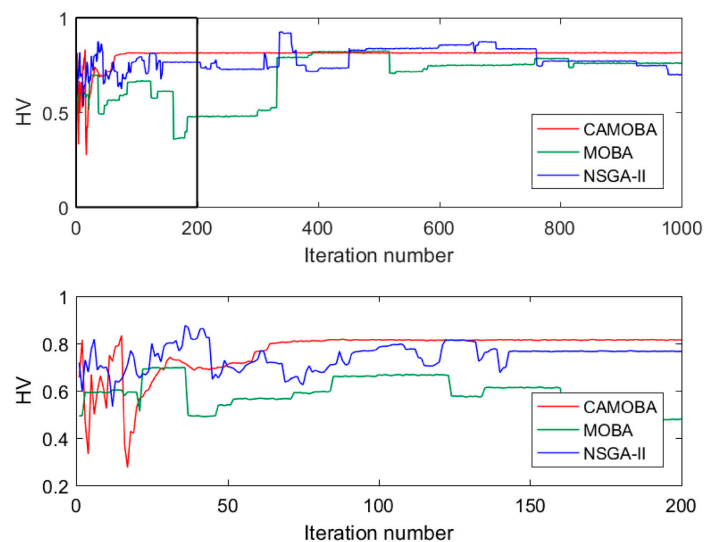

(f)
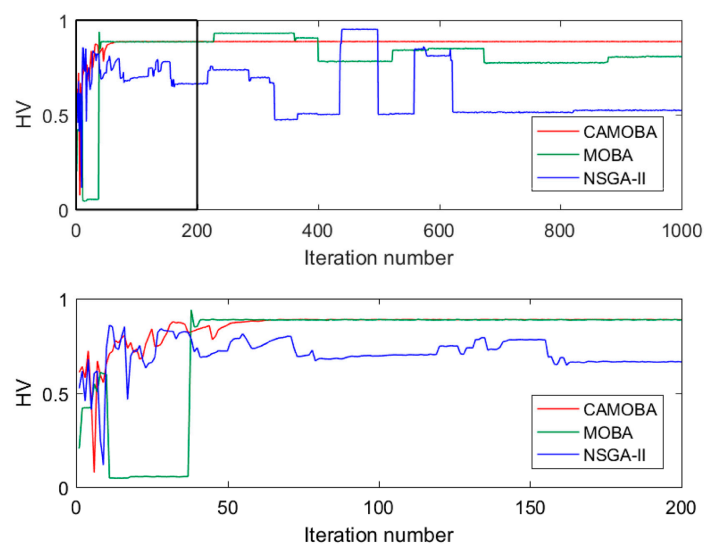

Figure 6. SP $(\mathrm{a}, \mathrm{c}, \mathrm{e})$ and $\mathrm{HV}(\mathrm{b}, \mathrm{d}, \mathrm{f})$ convergence curves of three algorithms in different hydrological years during iteration 1 to 1000 (first row) and 1 to 200 (second row): (a,b) wet year; (c,d) normal year; and $(\mathbf{e}, \mathbf{f})$ dry year. 

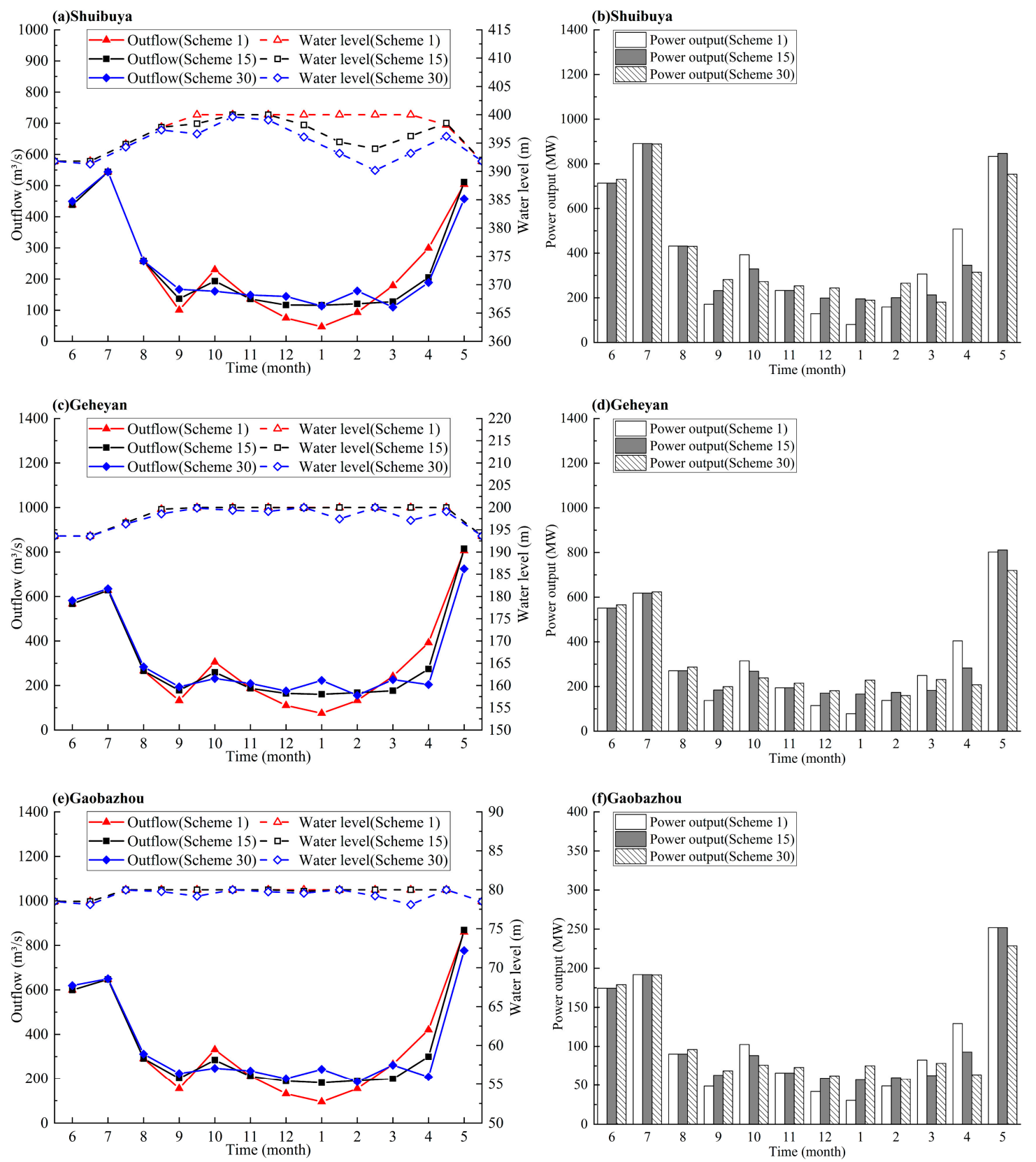

Figure 7. Reservoir operation processes obtained by CAMOBA in a normal year: $(\mathbf{a}, \mathbf{b})$ Shuibuya Reservoir; (c,d) Geheyan Reservoir; and (e,f) Gaobazhou Reservoir.

\section{Conclusions}

The $\mathrm{CHRO}$ is a complicated multi-objective optimization problem that simultaneously considers annual hydropower generation and firm power output. In this paper, CAMOBA has been proposed to handle the CHRO problem. In CAMOBA, the external archive set is added to preserve the non-dominant solutions. The population initialization based on chaos theory is adopted to improve population diversity. The self-adaptive local search strategy based on the normal cloud model is proposed to update solutions. The mutation operation is designed to mitigate premature convergence. Finally, CAMOBA is applied to the CHRO problem of the Qingjiang cascade hydropower stations in southern China. The case verifies the validity and feasibility of the proposed CAMOBA method. Its superiority in convergence and diversity is verified by comparing the results with other algorithms, including MOBA and NSGA-II. The experimental results indicate that there is a competitive relationship between the two targets in wet, normal and dry years. Compared to the MOBA and NSGA-II, the CAMOBA 
method proposed in this paper can generate more annual power generation with the same firm power output within a reasonable computational time, so it provides a new approach for solving the CHRO problem.

Author Contributions: Conceptualization, L.S. and K.Y.; methodology, L.S., K.Y. and H.H.; software, L.S.; validation, H.H. and K.Y.; formal analysis, L.S. and Z.Y.; data curation, K.Y.; writing-original draft preparation, L.S.; writing-review and editing, K.Y. and H.H.; supervision, Z.Y.

Funding: This research was funded by National Key Basic Research Program of China (973 Program) (2012CB417006).

Acknowledgments: This research was supported by National Key Basic Research Program of China (973 Program), grant number 2012CB417006.

Conflicts of Interest: The authors declare no conflict of interest.

\section{References}

1. Feng, Z.-K.; Niu, W.-J.; Cheng, C.-T. Optimizing electrical power production of hydropower system by uniform progressive optimality algorithm based on two-stage search mechanism and uniform design. $J$. Clean Prod. 2018, 190, 432-442. [CrossRef]

2. Madani, K.; Lund, J.R. Modeling California's high-elevation hydropower systems in energy units. Water Resour. Res. 2009, 45. [CrossRef]

3. Madani, K. Hydropower licensing and climate change: Insights from cooperative game theory. Adv. Water Resour. 2011, 34, 174-183. [CrossRef]

4. Niu, W.-J.; Feng, Z.-K.; Cheng, C.-T.; Wu, X.-Y. A parallel multi-objective particle swarm optimization for cascade hydropower reservoir operation in southwest China. Appl. Soft Comput. 2018, 70, 562-575. [CrossRef]

5. Reshma, T.; Reddy, K.V.; Pratap, D.; Agilan, V. Parameters Optimization using Fuzzy Rule Based Multi-Objective Genetic Algorithm for an Event Based Rainfall-Runoff Model. Water Resour. Manag. 2018, 32, 1501-1516. [CrossRef]

6. Reddy, M.J.; Kumar, D.N. Optimal Reservoir Operation Using Multi-Objective Evolutionary Algorithm. Water Resour. Manag. 2006, 20, 861-878. [CrossRef]

7. Wang, Y.; Hua, Z.; Wang, L. Parameter Estimation of Water Quality Models Using an Improved Multi-Objective Particle Swarm Optimization. Water 2018, 10, 32. [CrossRef]

8. Sivasubramani, S.; Swarup, K.S. Multi-objective harmony search algorithm for optimal power flow problem. Int. J. Electr. Power 2011, 33, 745-752. [CrossRef]

9. Hajibandeh, E.; Nazif, S. Pressure Zoning Approach for Leak Detection in Water Distribution Systems Based on a Multi Objective Ant Colony Optimization. Water Resour. Manag. 2018, 32, 2287-2300. [CrossRef]

10. Sun, X.; Luo, J.; Xie, J. Multi-Objective Optimization for Reservoir Operation Considering Water Diversion and Power Generation Objectives. Water 2018, 10, 1540. [CrossRef]

11. Li, F.F.; Qiu, J. Multi-Objective Reservoir Optimization Balancing Energy Generation and Firm Power. Energies 2015, 8, 6962-6976. [CrossRef]

12. Yang, X.-S. A new metaheuristic bat-inspired algorithm. In Nature Inspired Cooperative Strategies for Optimization (NISCO 2010); Cruz, C., González, J.R., Krasnogor, N., Pelta, D.A., Terrazas, G., Eds.; Springer: Berlin, Germany, 2010; pp. 65-74.

13. Bahmani-Firouzi, B.; Azizipanah-Abarghooee, R. Optimal sizing of battery energy storage for micro-grid operation management using a new improved bat algorithm. Int. J. Electr. Power 2014, 56, 42-54. [CrossRef]

14. Zarei, A.; Mousavi, S.F.; Gordji, M.E.; Karami, H. Optimal Reservoir Operation Using Bat and Particle Swarm Algorithm and Game Theory Based on Optimal Water Allocation among Consumers. Water Resour. Manag. 2019, 33, 3071-3093. [CrossRef]

15. Sathya, M.R.; Mohamed Thameem Ansari, M. Load frequency control using Bat inspired algorithm based dual mode gain scheduling of PI controllers for interconnected power system. Int. J. Electr. Power. 2015, 64, 365-374. [CrossRef] 
16. Niknam, T.; Azizipanah-Abarghooee, R.; Zare, M.; Bahmani-Firouzi, B. Reserve Constrained Dynamic Environmental/Economic Dispatch: A New Multiobjective Self-Adaptive Learning Bat Algorithm. IEEE Syst. J. 2013, 7, 763-776. [CrossRef]

17. Yang, X.-S. Bat Algorithm for Multi-objective Optimisation. Int. J. Bio-Inspir. Comput. 2011, 3, $267-274$. [CrossRef]

18. Yang, N.-C.; Le, M.-D. Optimal design of passive power filters based on multi-objective bat algorithm and pareto front. Appl. Soft Comput. 2015, 35, 257-266. [CrossRef]

19. Liang, H.; Liu, Y.; Li, F.; Shen, Y. A multiobjective hybrid bat algorithm for combined economic/emission dispatch. Int. J. Electr. Power 2018, 101, 103-115. [CrossRef]

20. Bansal, S.; Singh, A.K.; Gupta, N. Optimal Golomb Ruler Sequences Generation for Optical WDM Systems: A Novel Parallel Hybrid Multi-objective Bat Algorithm. J. Inst. Eng. India Ser. B 2016, 98, 43-64. [CrossRef]

21. Prakash, S.; Trivedi, V.; Ramteke, M. An elitist non-dominated sorting bat algorithm NSBAT-II for multi-objective optimization of phthalic anhydride reactor. Int. J. Syst. Assur. Eng. Manag. 2016, 7, 299-315. [CrossRef]

22. Zitzler, E.; Thiele, L. Multiobjective evolutionary algorithms: A comparative case study and the Strength Pareto approach. IEEE Trans. Evolut. Comput. 1999, 3, 257-271. [CrossRef]

23. Deb, K.; Pratap, A.; Agarwal, S.; Meyarivan, T. A fast and elitist multiobjective genetic algorithm: NSGA-II. IEEE Trans. Evolut. Comput. 2002, 6, 182-197. [CrossRef]

24. Aydin, I.; Karakose, M.; Akin, E. Chaotic-based hybrid negative selection algorithm and its applications in fault and anomaly detection. Expert Syst. Appl. 2010, 37, 5285-5294. [CrossRef]

25. Parlitz, U.; Ergezinger, S. Robust communication based on chaotic spreading sequences. Phys. Lett. A 1994, 188, 146-150. [CrossRef]

26. Li, D.; Cheung, D.; Shi, X.; Ng, V. Uncertainty reasoning based on cloud models in controllers. Comput. Math. Appl. 1998, 35, 99-123. [CrossRef]

27. Li, R.; Jiang, Z.; Li, A.; Yu, S.; Ji, C. An improved shuffled frog leaping algorithm and its application in the optimization of cascade reservoir operation. Hydrol. Sci. J. 2019, 63, 2020-2034. [CrossRef]

28. Bekele, E.G.; Nicklow, J.W. Multi-objective automatic calibration of SWAT using NSGA-II. J. Hydrol. 2007, 341, 165-176. [CrossRef]

29. Kannan, S.; Baskar, S.; McCalley, J.D.; Murugan, P. Application of NSGA-II Algorithm to Generation Expansion Planning. IEEE Trans. Power Syst. 2009, 24, 454-461. [CrossRef]

30. Schott, J.R. Fault Tolerant Design Using Single and Multicriteria Genetic Algorithm Optimization. Master's Thesis, Massachusetts Institute of Technology, Cambridge, MA, USA, 1995.

31. Zitzler, E.; Deb, K.; Thiele, L. Comparison of multiobjective evolutionary algorithms: Empirical results. Evol. Comput. 2000, 8, 173-195. [CrossRef] 